
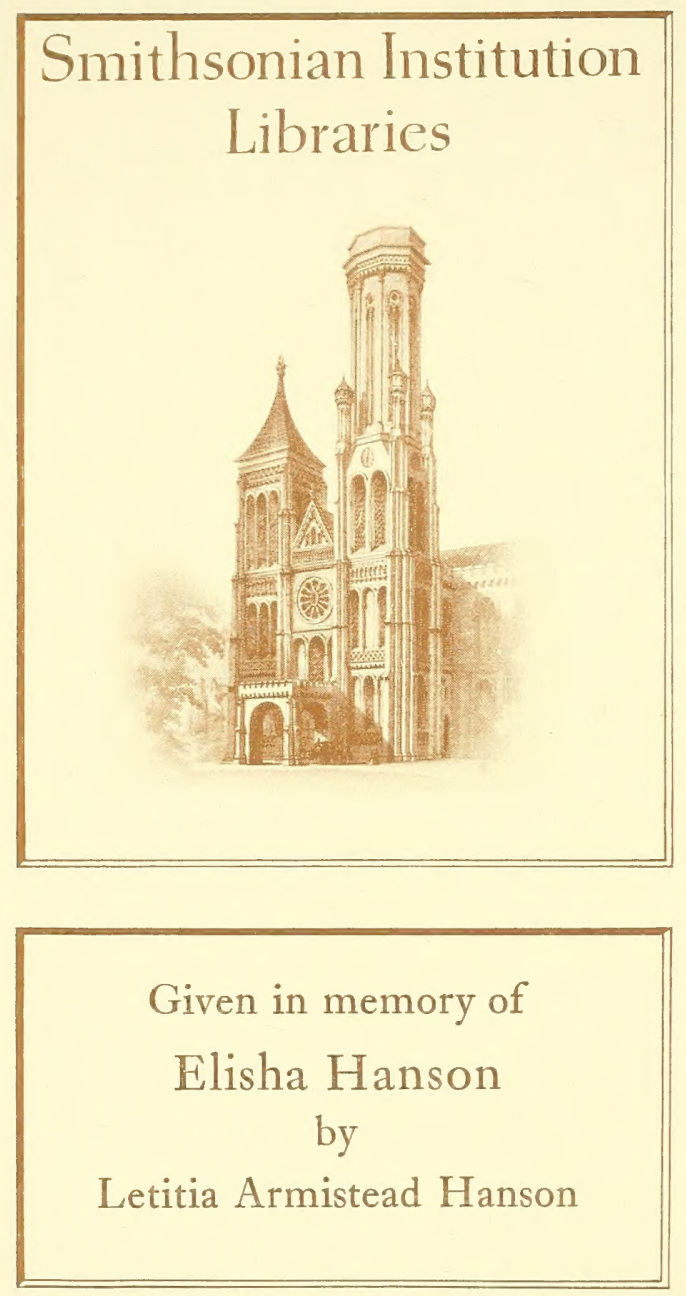



\title{
THE HISTORY OF SCIENCE AS AN ERROR BREEDER
}

\author{
By Professor G. A. MILLER \\ UNIVERSITY OF ILLINOIS
}

$W^{1}$

HILE the rôle of the history of science as an error exterminator is much more significant than its rôle as an error breeder, it is necessary to consider it in both of these lights in order to understand its bearings completely. If a comparatively harmless pest destroys pests which are more harmful than itself, it is desirable to consider the injuries which it inflicts as well as the services which it renders. The services that the history of science renders as an error exterminator have received much attention, especially in recent years, but its mischievous rôle as an error breeder seems to have received only little public notice, hence it may be profitable to consider here some striking evidences of this rôle.

In these days of great specialization in science, it is scarcely necessary to emphasize Sir W. Hamilton's dictum "the greater the extension the smaller the intension." All scientists are aware of the fact that it is a very difficult matter to secure a deep and satisfactory intellectual penetration even in a comparatively small domain of science. If one desires to obtain a comprehensive view of the fundamental developments in a larger domain, such as chemistry or mathematics, one has to take much for granted that has been said by others but has not been fully verified by oneself. This method of procedure has to be followed still more by those who strive to secure comprehensive views as regards the fundamental developments in science as a whole.

The highly commendable attitude of mind which seeks to understand the broad lessons taught by science as a whole and to secure a comprehensive view not only of the scientific work now being done in various countries but also of the work done during the preceding ages presents a great contrast when compared with that actuating the extreme specialist when working in a field which he has made his own. It is true that the historian of science is not always actuated by the former attitude of mind. He, too, has his special problems and ponders profoundly over some of the elements in his work. As regards these particular elements he stands on a par with the specialists in other fields.

The historian of science is compelled, however, when he is called upon to treat science as a whole, or even a large domain thereof, to take much for granted which he cannot verify on account of lack of 
time. It is here where the breeding of errors makes its appearance. The historian is greatly tempted to state interesting and striking facts. He finds that many such facts have been emphasized by the specialists, but he naturally fails at times in his efforts to interpret the language of the specialists. The next historian who tries to interpret the words of this earlier historian frequently misses the correct interpretaion still more, and hence statements conveying an entirely false notion tend to creep into the general histories of science.

As pure mathematics is the most exact science it may perhaps be assumed that the history of this subject is the most accurate among the histories of the various sciences. At any rate, it seems desirable to illustrate some of the preceding observations by examples of such an elementary type that they can be easily understood by all. The history of mathematics furnishes many such examples since some of its early permanent developments belong to a period when the mathematical specialists dealt with questions which all could easily understand. These specialists had not then raised themselves to great intellectual heights by standing on the shoulders of other specialists, who, in turn, stood on the shoulders of earlier specialists in almost endless succession.

To furnish a striking but somewhat extreme illustration of the fact that the mathematical historian is apt to repeat statements which he does not fully understand, it may be noted here that on page 165 of the third edition of Cantor's well-known Vorlesungen über Geschichte der Mathematik, 1907, it is stated that the Greeks used the term epimorion to denote the ratio $n /(n+1)$, and that "Archytas had already stated and proved the theorem that if an epimorion, $a / \beta$ is reduced to its lowest terms, which may be called $\mu / \nu$, then $\nu=\mu+1$. It is evident that this remark is practically meaningless, for if $a / \beta$ is an epimorion then it is obviously already in its lowest terms according to the definition of the term epimorion just noted, which seems itself to be incorrect. Notwithstanding this obvious lack of clearness, the statement appears again on page 53 of the second edition of Cajori's History of Mathematics, 1919, in spite of the fact that G. Eneström had in the meantime directed attention to its inaccuracy in volume 8 of the Bibliotheca Mathematica, 1917-1918, page 174.

An important feature of the history of science is that many statements made therein are intended to be true only in a general way, while others are supposed to be exact, and the reader has frequently to decide for himself to which of these two classes a particular statement is supposed to belong. For instance, one can usually not determine accurately who was the founder of a large subject since steps towards its development were commonly taken by a number of different men. On the other hand, such statements as "Newton and Leibniz were 
the founders of the calculus" have an important historical significance. One might almost say that such incomplete assertions constitute the meat of a general history of science. When it is remembered that they are supposed to represent only first approximations to the truth they can not be regarded in the light of actual error breeders, even if they are apt to have this effect temporarily, especially on beginners.

The significance of many statements in the history of science grows with the growth of the reader's breadth of knowledge relating to the subject involved. Statements which at one period of his scientific development would have appeared satisfactory may not appear so at a later period. It is, of course, impossible for an author of a general history of science to provide for these various stages of development, but there are instances where such authors have failed to provide a satisfactory account for any of their readers irrespective of the stage of their scientific development. While such instances are comparatively rare and do not necessarily impair seriously the works in which they appear they may serve to illustrate the general topic under consideration and are unusually interesting in themselves. Hence we give here one such instance relating to mathematics during medieval times.

On page 314 of Hankel's Geschichte der Mathematik, 1874, it is stated that "the first mathematical paper of the Middle Ages which deserves this name is a letter of Gerbert to Adelbold, bishop of Utrecht.". In view of the fact that we are now living in an age of numerous scientific papers, this remark by Hankel should be of great interest at the present time. Such interest is reflected in the fact that F. Cajori quotes this remark in the two editions of his History of Mathematics, 1894 and 1919, respectively, as well as in the two editions of his History of Elementary Mathematics, 1896 and 1917, respectively. Hence the American reader of the history of science is seriously exposed to the danger of assuming that the said remark by Hankel represents a well-established historical fact.

Such an assumption does not imply that the said letter by Gerbert, who died as Pope Sylvester II in 1003, contained any new mathematical results, since there are now expository mathematical papers as well as research papers. It does not imply that the letter in question was long, since there are now many brief mathematical papers as well as long ones. It does, however, imply that this letter was superior to the many other mathematical writings which had appeared during the four or five centuries which had then elapsed since the beginning of the Middle Ages.

It is true that such a superiority even in so long a period of years does not imply very much, since this particular period was unusually barren as regards mathematical developments. Notwithstanding this comparative barrenness, it includes a few noteworthy oases created 
especially by the Arabs in algebra and in trigonometry. In particular, the work from which our modern term algebra is derived was composed during this period, and the work of several well-known Hindu writers appeared therein. Hence the reader seems justified for having somewhat high expectations as regards the mathematical importance of Gerbert's letter if it actually deserves being called "the first mathematical paper of the Middle Ages." In fact, the non-mathematical reader might be inclined to fear that the mathematical merits of this letter were too great to lie within the limits of his comprehension.

These expectations and fears are apt to be enhanced by the reading of the accounts of Gerbert's letter in some of our most popular histories of mathematics, including the ones already noted. Not only is it stated here that this letter contained a correct explanation for the difference of the results obtained by using two different formulas for the determination of the area of an equilateral triangle, but some of the other statements relating to this letter are sufficiently obscure and misleading to arouse the suspicion that the subject treated therein might possibly be difficult. In various instances the obscurity is increased by the fact that figures of triangles which are not equilateral are given, while the text relates to an equilateral triangle. This is done, for instance, on page 249 of Günther's, Geschichte der Mathematik, 1908, as well as in the three editions of volume I of Cantor's well known Vorlesungen über Geschichte der Mathematik, pages 744, 815 and 866, respectively.

From the preceding remarks the reader will naturally conclude that the present writer does not believe that the letter in question merits to be called "the first mathematical paper of the Middle Ages which deserves this name," notwithstanding the fact that this epithet has been applied to it by eminent authoritities. In fact, the present writer believes not only that the letter does not merit this epithet but that it is of so little mathematical importance as to make it appear ridiculous to make such a claim for it. Moreover, he believes that other statements made about this letter in well-known mathematical histories are strikingly inaccurate. In order to establish the correctness of this point of view, it is necessary to state just what is found in the part of this letter which has been preserved, upon which our view of its merits must be based.

In view of the great claims made for this letter, the reader will naturally be surprised to find that it deals with the very elementary question of finding the area of an equilateral triangle, a question which had been completely solved many centuries before. Gerbert gives here the rule that the altitude of such a triangle can be found by subtracting one-seventh from its side, which is a sufficiently close approximation for many purposes, since the altitude is a $/ 2 \sqrt{ } 3$, where $a$ is the 
side. He then states, in substances, that the area of an equilateral triangle is one-half of the product of the base and the altitude thus obtained, and he calls this the "geometric rule" for finding such an area.

Thus far there is nothing surprising in this letter, and no one seems to have claimed much credit for this part, but Gerbert then makes some very inaccurate and foolish remarks about finding the area of such a triangle by another rule, called the "arithmetic rule," and it is just upon these remarks which exhibit a great lack of geometric insight that the high claims of this letter have been based. The inaccuracy of these remarks had been noted by $M$. Chasles in his well and favorably known Aperçu Historique, 1875, page 506, but notwithstanding this fact various later mathematical authors, including all those noted above, have called them correct in their general histories.

In order to appreciate the crudeness of this "arithmetic rule," which is equivalent to the formula $1 / 2 a(a+1)$, it may be noted that in the work of the Egyptian Ahmes, written about 1700 B. C., the area of an isosceles triangle seems to have been found by multiplying onehalf the base by a side instead of by the altitude. This method has been regarded as remarkable on account of its crudity, but when we are told that more than two thousand years later the Roman surveyors were taught to find the area of such a triangle by finding the product of one-half of the numerical measure of the base and a number which is even larger than the numerical measure of another side, there seems to be sufficient ground for surprise even in a scientific matter.

It must be admitted that the instances cited above are insufficient to establish the fact that a general history of science is an unusually favorable ground for the breeding and the propagation of scientific errors. In fact, all that has been attempted here is to advance a few reasons why one might suspect danger here, and to support these reasons by illustrations which were assumed to be also of interest to the reader on account of their unusual intrinsic. features. Perhaps a more conclusive argument in support of the thesis in question is furnished by the fact that G. Eneström noted more than two thousand desirable changes relating to the general history of mathematics by M. Cantor, to which reference was made. 



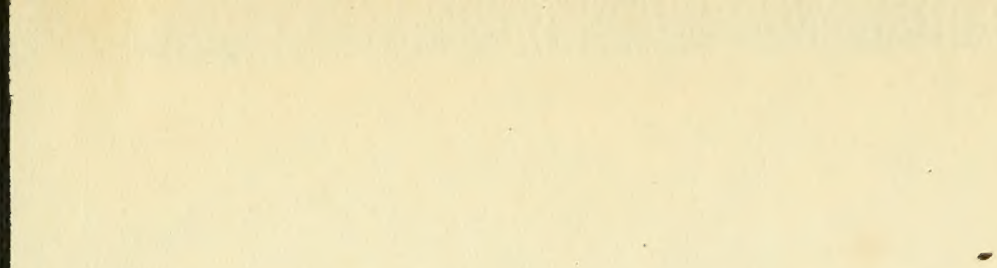


SMITHSONIAN INSTITUTION LIBRARIES

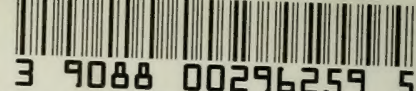

nhbird SF465.S56 59

Published figures and plates of the exti 